\title{
Social Sensors and Pervasive Services: Approaches and Perspectives
}

\author{
Alberto Rosi ${ }^{1}$, Simon Dobson ${ }^{2}$, Marco Mamei ${ }^{1}$, Graeme Stevenson ${ }^{2}$, Juan $\mathrm{Ye}^{2}$, Franco Zambonelli ${ }^{1}$ \\ 1) Department of Science and Engineering Methods, University of Modena and Reggio Emilia, Italy \\ 2) School of Computer Science, University of St Andrews, UK
}

\begin{abstract}
Social networks are perhaps the purest example of "Web 2.0" services, and offer a sophisticated tool for accessing the preferences and properties of individuals and groups. Thus, they potentially allow up-to-date, richly annotated contextual data to be acquired as a side effect of users' everyday use of the services. In this paper, we explore how such "social sensing" could be integrated into pervasive systems. We frame and survey the possible approaches to such an integration, and eventually discuss the open issues and challenges facing researchers.
\end{abstract}

\section{Introduction}

Social networks are popular means of information sharing among groups of similar interests. Every day social communities (as Facebook, Twitter, MySpace, LinkedIn, Orkut, etc.) attract millions of users and absorb from them detailed contextual information describing individual interests, preferences, and activities. Accordingly, social networks have the potential to act as sorts social sensing devices, providing us the opportunity of accessing massive amounts of information hard to be obtained via other means.

As of today, while many successful applications exploiting in this way social network tools already exist $[2,6]$, their exploitation as social sensors in the context of pervasive services and applications is still at an early stage. Indeed, bringing together the world of pervasive services and applications and the world of social networks and social sensing opens up several opportunities.

The ability of social sensing in the context of pervasive services scenario, thus coupling social sensing with the traditional sensing means of pervasive computing, makes it possible to provide pervasive applications with much higher degrees of context-awareness, further shortening the gap between digital and physical worlds and overall providing a much better service to users.

In this paper, the issue of integrating social sensing and pervasive computing is investigated with the aim of providing the following contributions:
- Identifying the potentials of social sensing, emphasizing its peculiarities with respect to more traditional means of sensing, and overviewing some (non pervasive) successful stories of social sensing exploitation.

- Analyzing and classifying the different architectural approaches that can be adopted to integrate social sensing and pervasive computing. We show that it is not only possible to exploit social networking tools as sources of social data for pervasive infrastructures, but also to adopt them as infrastructures to facilitate the integration of pervasive and social sensing.

- Surveying, according to the proposed classification, a number of representative proposals and applications from the literature.

- Introducing and analyzing a number of challenging research issues to be faced towards the systematic exploitation of social sensing in pervasive computing.

\section{Social Sensors: Potentials and Applications}

Pervasive computing scenarios generally consider an environment densely enriched with ICT-enabled sensorial capabilities, to be exploited for the provisioning of contextaware, adaptable, and personalized services for better interacting with the surrounding world.

Modern cities are already provided of cameras and microphones for security purposes, and sensor networks for environmental monitoring. RFID-based tickets and badges can keep track of user movements and activities. Smart phones are embedded with gyroscope, compass, accelerometer, proximity sensors, and localization tools. Thus, wrapping and parsing all the above measures is the first step toward building effective pervasive services.

However, it's beyond any doubts that the capabilities of social sensing can further enrich the scenario, by complementing the available information and thus enabling higher levels of context-awareness.

\subsection{Social Sensors vs. Pervasive Sensors}

With the term social sensor we intend any source of information that can be identified in modern social network- 
ing and Web tools expressing some situation and fact about users (e.g., their preferences or scheduled activities) and their social environment. Explicit examples of social sensors include Twitter posts, Facebook status updates, or pictures posted on Flickr. However, even what a user searches on google or what it buys online can be implicitly considered as a sort of social sensor, if used to that purpose.

Social sensors thus distinguish from pervasive sensors, intended as sources of information by ICT devices.

Of course, we are aware that pervasive sensors can sometimes act as social sensors too, i.e., they can be used to detect social facts as well as social networking tools can. For instance, the detection of friendship between two persons can be either from proximity sensors or achieved by mining their Facebook network. Nevertheless, the availability of multiple data sources can be of help to better support the assessment of situations.

In any case, the potential of social sensors can go much beyond that, since there are situations and facts that:

- Exist only in users' mind (e.g., a user likes a particular movie) and cannot be sensed by other pervasive sensing means, but only in the case such states of mind are reflected in social networking sites.

- Are revealed by social sensors and could be potentially revealed also by pervasive sensing, but simply happen to occur in their absence (i.e., a user posting a geotagged picture on Flickr can reveal the location of the user even in absence of localization sensors).

- Express information about future situations (e.g., reading a shared calendar or the Facebook status of a user) that would otherwise hard (e.g., via inference on historical data) or simply impossible to obtain.

It is important to note that social sensing has the same issues of accuracy and timeliness as other sensors - indeed perhaps more so, since (for example) sensing using Facebook or a diary requires that the user keeps her Facebook/diary page up to date in the normal course of events, which many people do not do, or do only inconsistently.

\subsection{Current (Non Pervasive) Social Sensing Apps}

Beside their application in pervasive computing scenarios, social sensors have already been recognized as a powerful tool to detect and predict collective patterns of behavior [2], possibly associated to events occurring in the real world [16]. Indeed, several experimental and commercial systems exist, where parsing the web for users' social information can be used to support commercial, demographic, and emergency management activities.

As for implicit social sensing is concerned, e-busines sites (like Amazon [29]) are analyzing behavior of their costumers in buying, in the attempt of properly recommending items or products. Likewise, Google Trends (http://www.google.com/trends), by using google searches as a sensor, can be very effective in measuring social and commercial trends [5].

As for social networking tools and explicit social sensing is concerned, current works and experimentations focus on the most popular tools such as Flickr, Twitter, and Facebook, due to the critical mass of information that can be extracted from them.

In [7], the authors present techniques to automatically identify the location of higher interests all over the world, by analyzing the spatial distribution of millions of geotagged pictures posted on Flickr. Results are in accord with commonsense opinions and with travel books suggestions.

In [23], it is demonstrated that Twitter, thanks to its real-time nature, could effectively act as a seismometer for the detection of earthquakes, simply by observing user tweets. More in general, the ability of identifying global trends and events by Twitter is at the base of numerous Twitter-based applications, such as Tweettronics (http://www.tweettronics.com/), oriented to identify market trends and brand awareness for marketing purposes.

Facebook is often cited for studies on network evolution and peer (as nodes of a graph) behavior. Between the many examples of services extracting social features from a network, we mention [11] that studies and analyses the patterns of friend-making, and the work of [26] that studies the dynamic properties of the friendship network. Both of these works have the potential of reaching a better understanding of the dynamics of real-world social networks (and, therefore, potentially of highly commercial and social impact).

\subsection{Towards Pervasive Social Sensing Apps}

The above examples of social sensing applications focus on large-scale statistical collective behaviors, and are not specifically aimed at exploiting the knowledge extracted for the sake of improving pervasive services (or deploying innovative ones). Nevertheless, there are many signals that the trend towards the integration of the social networking and the pervasive computing world is in act.

Both Facebook and Twitter now integrate the possibility of automatically geo-locating users and posts, which is somehow a form of integration between pervasive sensors (the GPS) and social ones (the posts themselves). Other social networking tools are emerging that are explicitly conceived to facilitate collecting sensorial information for pervasive usage. For instance, in the area of traffic detection, Traffic AUS (http://itunes.apple.com/au/app/austraffic/) and Waze (http://world.waze.com/) propose social networks for car drivers, in which the data produced by drivers about the traffic situation can be exploited by other drivers for real-time navigation. 


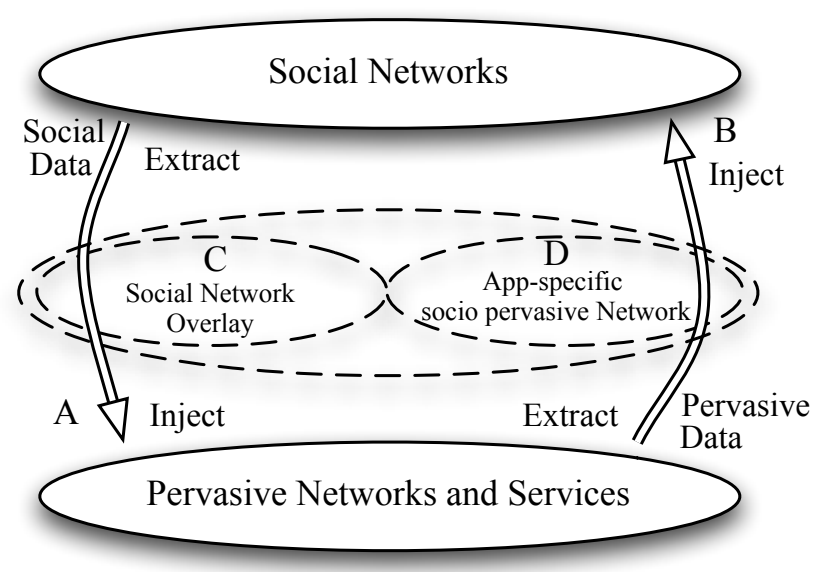

Figure 1. Approaches to integrate social
sensing and pervasive services.

However, the road towards the extensive and systematic integration of pervasive services and sensors with social sensors is far to come. Yet, a great deal of research activity exists in this directions, also showing that such integration can occur in many diverse ways.

\section{Classification and Survey of Approaches}

We have studied and analyzed a large body of proposals related to the integration of social and pervasive sensing and specifically aimed at improving and/or facilitating the development of pervasive services. On this basis, we have identified and framed four key ways by which such integration can be architected and pursued (see Fig. 1).

First, one can exploit social networks to extract social information from them, and have such information feeding pervasive services and applications (arrow $A$ in Fig.1), thus bringing social sensors at the same network level of pervasive sensors. This way of approaching the integration is the one that more directly reflects our own research efforts [15]. Related proposals are analyzed in Section 3.1.

Second, and somewhat reversing the former one, one can exploit social network tools (the place in which social sensing reside) also as a way to collect and organize the data coming from pervasive sensors (arrow $B$ in Fig.1). In this way, pervasive sensors are brought up at the same level of social sensors, and there integrated with them. Possibly even more important, in this way of approaching the integration, the social network infrastructure is elevated to the role of middleware for pervasive services and applications, i.e., as a socio-pervasive medium to distribute and fetch pervasive content and information. Proposals in this direction are analyzed in Section 3.2.
Third, and alternative to the second one, one could think of exploiting existing social network infrastructures not "as they are", but rather as the ground upon which to build an overlay in which to perform the integration (oval $C$ in Fig.1). In this way, the existing functionalities (e.g., for information diffusion and distribution or for event notification) of the social network infrastructure can be properly extended to account for the specific needs of pervasive sensors too. Related proposals are analyzed in Section 3.3.

A fourth way of approaching the integration, somewhat pushing the third one to the extreme, considers creating brand new socio-pervasive infrastructures, typically on an application-specific basis (oval $D$ in Fig.1). Such sociopervasive infrastructures thus act as the medium in which integration between the social and the pervasive sensors take place. Proposals in this direction are analyzed in Section 3.4.

\subsection{Extracting Data from Social Networks}

As stated above, some proposals focus on extracting information from social networks and digesting them to produce knowledge that can be eventually exploited in pervasive services, as if such social sensing knowledge were at the same level of pervasive sensing one.

Fujisaka et al. [10] propose methods for the detection of unusual crowding in physical locations from existing blog community. Here authors, by the analysis of common patterns of occurrence in each region over a specified time period, achieve the extraction of useful and interesting movement patterns, reflecting the occurrence of critical events in a geographic region. Experimental evaluation of the proposed method uses a real dataset collected from Twitter.

R. Ji et al. [12] report a work on mining famous city landmarks from blogs for personalized tourist suggestions. Their main contribution is a graph modeling framework to discover city landmarks by mining blog photo correlations with community supervision.

Q. Zhao et al. [28] propose detecting and framing events from the real world by exploiting the tags supplied by users in Flickr photos. The temporal and locational distributions of tag usage are analyzed, tags related to aperiodic events and those of periodic events are distinguished. Tags are finally clustered and, for each cluster, a representing picture and tag is extracted.

T. Lovett et al. [14] present two heuristic methods for data fusion that combine the user's personal calendar with social network posts, in order to produce a real-time multisensor interpretation of the real-world events. This study shows that the calendar can be significantly improved as a sensor and indexer of real-world events through data fusion.

From our side, we have developed an unattended system [15] able to extract and take advantage of up-to-date and spontaneous information embedded with pictures. With ex- 
periments on the Flickr database, we have shown that this system, by learning from past touristic user experience, is able to make effective recommendations to people visiting touristic places for the first time.

\subsection{Exploiting Social Networks as a Socio- pervasive Middleware}

Proposals in this area consider social network infrastructures as a sort of socio-pervasive middleware in which to make converging and conciliating data from different sources, and specifically pervasive sensors, and of which to exploit the functionalities for data and event management.

M. Demirbas et al. [8] have designed and implemented a crowd-sourced sensing and collaboration service over Twitter, for two application scenarios: a crowd-sourced weather radar, and a participatory noise-mapping application. The whole system is based on the intuition of exploiting of Twitter as a publish-subscribe for the storing and the diffusion of information and events about pervasive sensors and userprovided sensing data.

S-Sensors [3] provides a framework to globally share locally measured sensory readings. This paper proposes to employ micro-blogging to publish and share sensory data and resources, where short messages depicting the status of the environment are used to convey sensory data of the physical world. Here, sensor networks may utilize social network tools to distribute the sensing responsibilities amongst the networks.

D.Patterson et al. [18] present a prototype system that automatically infers users' place, activity, and availability from sensors on their handheld devices or laptop computers. Data is then reported to buddies through embedding information in commercial instant-messaging profile status.

\subsection{Pervasive Overlays on Social Networks}

Proposals in this area are related to interconnecting and sharing data sensed from personal devices with the rest of the world. Accordingly, overlays over existing social networks infrastructure are realized to interfacing such local networks and, to accomplish with peculiar application requirements, to implement or extend existing functionalities.

SenseFace [19] is a software overlay suitable for capturing the sensory data produced from user personal devices, processing and storing the sensory data in his/her personal gateway (which is a mobile device) and sending the data to a remote Internet gateway. Finally, disseminating the sensory data is demanded to a list of his/her social networks.

Z. Anwar et al. [1] propose an overlay constructed on top of the Orkut social network. Their aim is to demonstrate that an alternative model to query the social network, where each node chooses its peers to query using metrics that can account for data coming from pervasive sensors, not only improves the overall search time but also gives a sizable improvement in lookups, average round-trip delay and scalability.

M. Rahman et al.[20] propose a framework that bridges body sensor networks (BSNs) and social networks by mapping a subgroup of members of one's social network with each sensory data feed of his BSN. Thus, any sensor triggering sensory data, push from a BSN, is directly forwarded to the interested subgroup of one's social network only.

M. Rahman et al. [21] present a context-aware social network framework that can dynamically mash-up user sensory data and social networks. SenseFace provides facilities to push sensory data collected from user personal devices to the members of social networks, further providing realtime query of any sensory data by any authorized member of each social networks.

\subsection{App-specific Socio-pervasive Networks}

Here, the common idea of the analyzed proposals is to create application-specific network infrastructures (typically through mobile ad-hoc networks), and related logical layers, as the locus in which socio-pervasive information exists and is made available for specific pervasive services.

Automated Murmurs [9] represents a mobile platform which leverages the popularity of mobile and social computing to produce a location-sensitive messaging system which delivers user generated content to the public on the basis of both physical location and social relations.

A. Beach et al. [4] propose a system called SocialFusion for fusing mobile computing, social networks, and user personal sensors to promote socially-aware diffusion of information and events. A multi-stage architecture is proposed, in which issues of collecting and managing diverse data streams, mining the data for context-aware inferences of individuals and groups, and preserving privacy and anonymity, are addressed.

D.O.Olgun et al. [17] present the design, implementation and deployment of a wearable social sensing platform that can measure and analyze personal and social behaviors in a variety of settings and applications. Individual and social patterns of behavior are identified measuring face-to-face interaction, conversational dynamics, physical proximity to other people, and physical activity levels.

The approach of integrating real-world data from faceto-face proximity with identities in on-line social networks, has been also followed by V. d. Broeck et al. [25] that have developed an application to for people attending to scientific conferences. Personal profiles of the participants were automatically generated using several Web 2.0 systems and semantic data sources, and integrated in real-time with faceto face proximity relations detected using RFID badges. 


\section{Challenges and Open Issues}

To effectively combine social sensors and pervasive services, several challenges need to be faced. On the one hand, turning social networks into plug-and-play social sensing devices for future pervasive computing applications definitely asks for a deep rethinking (in terms of architecture and role) of both social networks and pervasive infrastructures, possibly along the directions identified in Section 3. On the other hand, the use of the information coming from social network applications in pervasive services requires mechanisms to extract meaningful data, usable by applications and services (other than readily understandable by humans), from the myriad of raw facts and data produced by social and pervasive sensors.

The latter problem is a centerpiece in context-awareness and pervasive computing research, and it is further exacerbated by the presence of social sensors, due to their peculiar differences from ICT sensing devices: $(i)$ the data is often in free-text with no structure nor codified semantics, thus complex to process and understand; (ii) there could be no guarantee on the delivery of specific information about specific facts and at specific times by social sensors; (iii) social sensors are completely out of the control loop of system managers and application developers. In a sentence, the people behind social sensors can post whatever (even wrong) information in whatever format, or simply not post anything at all.

Accordingly, we identified a number of general challenging issues.

Modelling Framework. While current social services are application specific, future social services should be constructed on the basis of common and re-usable tools and mechanisms. In particular, what is still missing is a general modeling view to frame data together. This framework should be able to manage data's spatial and temporal dimensions, and allowing for context-aware services to operate upon. In this field, STEvent [13] represents an interesting model for the identification of events and situations happening between people of a community. Following the graph theory, they build a model for events extractions around the idea rather than events and situations about people can be derived from the graph-links among them.

Data reconciliation. Given the exploding amount of data about the world that will soon be made available by both pervasive computing technologies and participatory Web tools, better mechanisms to exploit and reconcile such data are to be developed. In particular, researchers will have to deal with a wide plethora of sensing devices committing measures at different rates and resolutions, accuracies to be valued and data format to be conciliated. Furthermore, sampled data may have overlaps and conflicts. What is still mainly missing here is a common ground of tools and al- gorithms for data reconciliation, to resolve uncertainty and composing with each single portion of context, a unitary and smooth vision on events. In this field, [27] proposes an interesting model of context uncertainty that represents semantics of context uncertainty and exhibits fine-grained approaches to evaluate and resolve uncertainty when processing and using context.

Unified data representation and interpretation. Dealing with events, locations, date, etc, cannot neglect a shared vocabulary (that is, the type of objects and/or concepts that exist, and their properties and relations) used to model a domain. This representation should avoid complex and highly-structured formats. Instead, the use of pragmatic (i.e., tag-based) ontologies to encode such diverse information could support both an effective creation of such descriptions and an effective use by applications [22]; their integration with shared vocabulary represents a challenge for future research. In addition, to better interpret such complex data, visualization techniques and tools should be developed. Data visualization tools can be the user interface to certain applications, and they could become a core asset to see and understand the data produced by social and pervasive sensors at multiple levels of granularity. Works like Situvis [6] can represent an important step in this direction.

Data critical mass Before social networks could widely contribute to sensing properties about people, they have to reach a critical mass of data wideness to bank on. For instance, in our experience on Flickr [15], only a restricted number of cities in the world already have enough information to make our tourist recommendation tool applicable. In general, for social networks to become sensing devices, the limit on the area of applicability should be overtaken. If from one side we could imagine a wider user adoption of social services in the next few years, from another side more efficient extraction tools need to be conceived.

Privacy concerns. Privacy-related challenges are cross cutting concerns that impact on the all above challenges (for example, you will not have data critical mass if users are uncomfortable in sharing it). These is a general problem related to pervasive computing that is even more important when taking social sensor into consideration. More in detail, despite the fact that people consciously agree both of providing their personal data and of been tracked by social networks services (e.g., many social networks are also proposing localization services), new rules for respecting and preserving overall user privacy have to be formulated. Aggregating and anonymizing data can be useful rough solutions [24], but more investigation will be needed to sort out privacy intricacies of future pervasive applications.

Solving the above challenges can allow a systematic integration of social sensors and pervasive services, and thus spur a wide range of novel applications. 


\section{Conclusions}

In this paper we argued the importance of exploiting existing social networks as sorts of social sensing devices. We framed and described four possible approaches to integrate social networks and pervasive networks (also surveying the relevant proposals in the area) and eventually discussed the open issues towards making such integration possible and easy to use.

Along the identified directions, our current research work in the context of the SAPERE (Self-aware Pervasive Service Ecosystems) project, is aimed at identifying general solutions for the exploitation of social sensing data in pervasive service infrastructures, to make services highly aware of their context of use and self-adaptive accordingly.

Acknowledgements: Work supported by the SAPERE project (EU FP7-FET, Contract No. 256873).

\section{References}

[1] Z. Anwar, W. Yurcik, V. Pandey, A. Shankar, I. Gupta, and R. H. Campbell. Leveraging social-network infrastructure to improve peer-to-peer overlay performance: Results from orkut. CoRR, abs/cs/0509095, 2005.

[2] S. Asur and B. A. Huberman. Predicting the future with social media. CoRR, abs/1003.5699, 2010.

[3] M. Baqer and A. Kamal. S-sensors: Integrating physical world inputs with social networks using wireless sensor networks. In ISSNIP Conference, pages 213 -218, 2009.

[4] A. Beach, M. Gartrell, X. Xing, R. Han, Q. Lv, S. Mishra, and K. Seada. Fusing mobile, sensor, and social data to fully enable context-aware computing. In HotMobile Workshop, pages 60-65, Annapolis (MR), 2010.

[5] H. Choi and H. Varian. Predicting the Present with Google Trends. Technical report, Google Inc., 2009.

[6] A. K. Clear, T. Holland, S. Dobson, A. Quigley, R. Shannon, and P. Nixon. Situvis: A sensor data analysis and abstraction tool for pervasive computing systems. Pervasive Mob. Comput., 6:575-589, Oct. 2010.

[7] D. J. Crandall, L. Backstrom, D. Huttenlocher, and J. Kleinberg. Mapping the world's photos. In WWW Conference, pages 761-770, Madrid (S), 2009.

[8] M. Demirbas, M. Bayir, C. Akcora, Y. Yilmaz, and H. Ferhatosmanoglu. Crowd-sourced sensing and collaboration using twitter. In WOWMOM Symposium, pages 1 -9, 2010.

[9] J. Freyne, A. Brennan, B. Smyth, D. Byrne, A. Smeaton, and G. Jones. Automated murmurs: The social mobile tourist application. In CSE Conference, volume 4, pages 1021 1026, 2009.

[10] T. Fujisaka, R. Lee, and K. Sumiya. Detection of unusually crowded places through micro-blogging sites. In IEEE AINA Workshops, pages $467-472,2010$.

[11] H. Hui-Yi and P. Hung-Yuan. Use behaviors and website experiences of facebook community. In ICEIE Conference, volume 1, pages V1-379-V1-383, 2010.

[12] R. Ji, X. Xie, H. Yao, and W.-Y. Ma. Mining city landmarks from blogs by graph modeling. In ACM Multimedia Conference, pages 105-114, Beijing (CH), 2009.
[13] H. W. Lauw, E.-P. Lim, H. Pang, and T.-T. Tan. Stevent: Spatio-temporal event model for social network discovery. ACM Trans. Inf. Syst., 28(3):1-32, 2010.

[14] T. Lovett, E. O'Neill, J. Irwin, and D. Pollington. The calendar as a sensor: analysis and improvement using data fusion with social networks and location. In ACM UBICOMP Conference, pages 3-12, New York, NY, USA, Sept. 2010.

[15] M. Mamei, A. Rosi, and F. Zambonelli. Automatic analysis of geotagged photos for intelligent tourist services. In Conference on Intelligent Environments, 2010.

[16] M. Mendoza, B. Poblete, and C. Castillo. Twitter Under Crisis: Can we trust what we RT? In Social Media Analytics Workshop, Washington (DC), July 2010.

[17] D. O. Olgun and A. S. Pentland. Social sensors for automatic data collection. In Americas Conference on Information Systems, 2008.

[18] D. Patterson, X. Ding, S. Kaufman, K. Liu, and A. Zaldivar. An ecosystem for learning and using sensor-driven im status messages. Pervasive Computing, IEEE, 8(4):42 -49, 2009.

[19] M. Rahman, A. El Saddik, and W. Gueaieb. Senseface: A sensor network overlay for social networks. In IEEE IMCT Conference, pages 1031 -1036, May 2009.

[20] M. Rahman, A. El Saddik, and W. Gueaieb. Building dynamic social network from sensory data feed. Instrumentation and Measurement, IEEE Transactions on, 59(5):1327 -1341, May 2010.

[21] M. Rahman, S. Hamdan, A. El Saddik, and W. Gueaieb. Context-aware social networks mashup: A personalized web perspective. In IEEE IMTC Conference, pages 1228 -1233, May 2010.

[22] V. Robu, H. Halpin, and H. Shepherd. Emergence of consensus and shared vocabularies in collaborative tagging systems. ACM Trans. Web, 3:14:1-14:34, September 2009.

[23] T. Sakaki, M. Okazaki, and Y. Matsuo. Earthquake shakes twitter users: real-time event detection by social sensors. In WWW Conference, pages 851-860, Raleigh (NC), 2010.

[24] J. Shi, R. Zhang, Y. Liu, and Y. Zhang. Prisense: Privacypreserving data aggregation in people-centric urban sensing systems. In IEEE INFOCOM Conference, 2010.

[25] W. Van den Broeck, C. Cattuto, A. Barrat, M. Szomszor, G. Correndo, and H. Alani. The live social semantics application: a platform for integrating face-to-face presence with on-line social networking. In PerCol Workshop at PerCom, pages 226-231, 2010.

[26] B. Viswanath, A. Mislove, M. Cha, and K. P. Gummadi. On the evolution of user interaction in facebook. In ACM WOSN Workshop, pages 37-42, Barcelona (S), 2009.

[27] J. Ye, S. McKeever, L. Coyle, S. Neely, and S. Dobson. Resolving uncertainty in context integration and abstraction: context integration and abstraction. In ACM ICPS Conference, pages 131-140, Sorrento (I), 2008.

[28] Q. Zhao, T.-Y. Liu, S. S. Bhowmick, and W.-Y. Ma. Event detection from evolution of click-through data. In $A C M$ KDDM Conference, pages 484-493, Philadelphia (PA), 2006.

[29] C.-N. Ziegler, G. Lausen, and J. A. Konstan. On exploiting classification taxonomies in recommender systems. $A I$ Commun., 21:97-125, April 2008. 